\title{
Glycoproteomic and glycomic databases
}

\author{
Deniz Baycin Hizal', Daniel Wolozny ${ }^{1}$, Joseph Colao', Elena Jacobson', Yuan Tian², Sharon S Krag ${ }^{3}$, \\ Michael J Betenbaugh ${ }^{1}$ and Hui Zhang ${ }^{2^{*}}$
}

\begin{abstract}
Protein glycosylation serves critical roles in the cellular and biological processes of many organisms. Aberrant glycosylation has been associated with many illnesses such as hereditary and chronic diseases like cancer, cardiovascular diseases, neurological disorders, and immunological disorders. Emerging mass spectrometry (MS) technologies that enable the high-throughput identification of glycoproteins and glycans have accelerated the analysis and made possible the creation of dynamic and expanding databases. Although glycosylation-related databases have been established by many laboratories and institutions, they are not yet widely known in the community. Our study reviews 15 different publicly available databases and identifies their key elements so that users can identify the most applicable platform for their analytical needs. These databases include biological information on the experimentally identified glycans and glycopeptides from various cells and organisms such as human, rat, mouse, fly and zebrafish. The features of these databases - 7 for glycoproteomic data, 6 for glycomic data, and 2 for glycan binding proteins are summarized including the enrichment techniques that are used for glycoproteome and glycan identification. Furthermore databases such as Unipep, GlycoFly, GlycoFish recently established by our group are introduced. The unique features of each database, such as the analytical methods used and bioinformatical tools available are summarized. This information will be a valuable resource for the glycobiology community as it presents the analytical methods and glycosylation related databases together in one compendium. It will also represent a step towards the desired long term goal of integrating the different databases of glycosylation in order to characterize and categorize glycoproteins and glycans better for biomedical research.
\end{abstract}

\section{Introduction}

Glycosylation is a critical protein modification relevant to numerous physiological functions and cellular pathways. It is important for protein folding, signaling and stability in the circulatory system $[1,2]$. Alterations in the glycosylation site occupancy or glycan structures of glycoproteins have been associated with hereditary and chronic diseases such as cancer, diabetes, cardiovascular, inflammatory, neurological and neuromuscular diseases [3-5]. Indeed, the fields of glycopathology and glycophysiology are providing a broader understanding of disease genesis and progression [6]. Furthermore, glycoproteins have been extensively studied for the discovery of disease associated modifications that can be used for both diagnosis and/or therapy for these diseases $[4,7]$.

Glycomics and glycoproteomics are two approaches used for the characterization of a specific cell, tissue or

\footnotetext{
* Correspondence: hzhang32@jhmi.edu

${ }^{2}$ Department of Pathology, Johns Hopkins University, Baltimore, MD, USA

Full list of author information is available at the end of the article
}

organ's glycoproteome and glycome from an extracted protein mixture in a specific state. The glycoproteome is the full composition of glycoproteins in a specific cell or tissue type, while the glycome is the full set of proteinbound sugar groups. Glycomics focuses on the study of glycan structure whereas glycoproteomics focuses on glycosylated proteins and glycosylation sites. In glycoproteomic analysis, glycosylated proteins are first enriched with proper analytical techniques and then analyzed by LC/ MS/MS for protein and glycosylation site identification. In glycomic analysis, the glycan moiety is often released from the glycoprotein and analyzed by mass spectrometry separately or in combination with chromatographic techniques. The chromatographic techniques can provide additional glycan identification and as well as the retention time of each identification. In addition, glycopeptides containing glycosylation sites and attached glycans can be analyzed by mass spectrometry without the release of glycans, which allows the identification of the glycosylation site and the specific glycans attached to the glycosylation site [8]. Initial works $[9,10]$ and recent reviews have 
extensively discussed analytical techniques used for identification and quantification of both the glycome and glycoproteome [4,11-15]. Programs have recently being initiated both to merge current methodologies for identification of glycans or glycoproteome from complex tissues or cells and to establish databases for the identified glycosylated proteins $[16,17]$. Although many of the publicly available databases are dynamic and updated, they are not being used effectively because of a lack of common resources, websites, and public awareness. Collating all of these databases is critically important to the glycobiology community since data analysis is another key element in addition to analytical methods. This review summarizes the conventional methodologies used in glycoproteomic and glycomic studies and also assembles 15 different glycosylation related databases for the scientific community. Furthermore, this manuscript also introduces three glycoproteomic databases developed by our group: UniPep [18], GlycoFly [19] and GlycoFish [20].

\section{Glycoproteomic databases}

Glycoproteomics is an emerging field which provides qualitative and quantitative information on a large number of glycoproteins. Recent improvements in glycoprotein isolation methods, bioinformatics, and mass spectrometry techniques have stimulated the subfield of proteomics known as glycoproteomic research [21].

In order to identify glycoproteins in a biological sample, the glycosylated proteins are first enriched with analytical, affinity, or chemical techniques. Subsequently, the type of glycosylation is determined. There are two major classes of glycosylation $\mathrm{N}$-glycosylation and $\mathrm{O}$-glycosylation.
With N-glycosylation, the glycan group is attached to usually N4 residues of asparagines, whereas in $\mathrm{O}$-glycosylation, the glycan group attaches to the hydroxyl oxygen of serine or threonine residues of a glycoprotein.

Emerging mass spectrometry techniques have significantly improved glycoproteomic studies. After the glycopeptides are enriched with a specific method, they can be qualitatively or quantitatively analyzed by tandem mass spectrometry to identify a large set of glycoproteins. A variety of technologies such as hydrazide chemistry, lectin chromatography or bead-immobilized techniques have been used for comprehensive analysis of site-specific glycosylation [22-26]. Although there are organized and structured databases for the proteomes and genomes of organisms which are complementary to each other, there is an absence of a unified, structured database for glycoproteome and glycome of organisms. Fortunately, a number of groups have established dynamic, publicly available databases to share their glycoprotome data $[18,27,28]$. Below are two tables, Tables 1 and 2 , listing many of the databases concerned primarily with glycoproteomics and glycomics.

\section{UniPep}

The detection and interpretation of the changes in organ and plasma proteomes may provide information and insights for delineating disease states. For this reason, it is important to discover serum or organ-specific biomarkers for early detection of the disease. Profiling the glycoproteome of plasma and organs is promising because changes in the pathological or physiological state of the human body can be manifested by aberrant glycosylation $[18,24]$.

Table 1 Summary of glycoproteomic databases

\begin{tabular}{|c|c|c|c|c|}
\hline Database & Type & Species & Method & Entries \\
\hline Unipep & $\begin{array}{l}\text { N-Glycosylated proteins } \\
\text { and peptides }\end{array}$ & Homo sapiens & $\begin{array}{l}\text { Hydrazide chemistry \& Solid Phase Extraction, in-silico } \\
\text { triptic digestion of IPI proteins, and prediction of NXS/T } \\
\text { glycosylation site with proteotypic potential }\end{array}$ & 2265 \\
\hline Glycofly & $\begin{array}{l}\text { N-Glycosylated proteins } \\
\text { and peptides }\end{array}$ & Drosophila melanogaster & Hydrazide chemistry and Solid Phase Extraction & 740 \\
\hline Glycofish & $\begin{array}{l}\text { N-Glycosylated proteins } \\
\text { and peptides }\end{array}$ & Danio Rerio & Hydrazide chemistry and Solid Phase Extraction & 269 \\
\hline GlycoSuiteDB & $\begin{array}{l}\text { O-linked and N-linked } \\
\text { Glycoproteins and glycans }\end{array}$ & & Published glycoproteins with different methods & 9436 \\
\hline GlycoProtDB & $\begin{array}{l}\text { N2 and mouse tissues } \\
\mathrm{N} \text {-Glycoproteins }\end{array}$ & $\begin{array}{l}\text { Caenorhabditis elegans } \\
\text { Mus Musculus }\end{array}$ & Lectin Concavilin A Chromatography & 1465 \\
\hline $\begin{array}{l}\text { O-GlycBase } \\
\text { NetOGlyc }\end{array}$ & $\begin{array}{l}O \text { and C-Glycosylated } \\
\text { proteins }\end{array}$ & $\begin{array}{l}\text { Combination of } \\
\text { references }\end{array}$ & $\begin{array}{l}\text { Data curation from literature and coupling ZFN gene } \\
\text { targeting, SimpleCell and Lectin Chromatography }\end{array}$ & $\begin{array}{l}2413 \\
3000\end{array}$ \\
\hline dbOGAP & O-GlcNAcylated proteins & $\begin{array}{c}\text { Homo Sapiens } \\
\text { Mus Musculus } \\
\text { Rattus Norvegicus } \\
\text { Drosophila Melanogaster } \\
\text { Xenopus Laevis }\end{array}$ & Curation from literature and SVM based prediction & $\begin{array}{l}798 \text { (exp) } \\
300 \text { (pred) }\end{array}$ \\
\hline
\end{tabular}


Table 2 Summary of glycomic databases

\begin{tabular}{lcr}
\hline Database & Type & Method \\
\hline (CFG) Glycan Structure DB & Glycan and glycan binding proteins & Glycan array screening, Glycan profiling \\
GlycoBase & $N$ and O-linked glycan structures & HPLC based and MS based glycan analysis \\
GlycomeDB & Carbohydrate structures & Carbohyrate data from CFG, KEGG, BCSDB, Carbbank \\
GlycoGeneDB & GlycoGenes and mRNA expression & In-silico collection of cloned and characterized human glycogenes \\
Glycan Mass Spectral DB & $N$-and O-linked glycans, and glycolipid glycans & $\begin{array}{c}\text { Glycan glycosidase digestion and analysis by HPLC with fluorescence } \\
\text { or MS }{ }^{n} \text { analysis }\end{array}$ \\
Lectin Frontier Database & Glycan-lectin interactions & Frontal affinity chromatography with fluorescence detection \\
\hline
\end{tabular}

Zhang et al. conducted a study to connect the organ and plasma proteomes using the hydrazide chemistry method to capture the $N$-glycosylated proteins [24] of plasma, bladder, breast cancer cells, liver, lymphocytes, cerebrospinal fluid, prostate tissue and prostate cancer cells [18]. In this study, 2265 unique $N$-linked glycosylation sites were identified with high confidence and these glycosylation sites and associated glycoproteins are publicly available within the UniPep website (www.unipep.org) [27]. In addition, thousands of unique $N$-linked glycosites from different mouse tissues were also reported [29-31]. The database for mouse $N$-linked glycosites can be developed using a similar process. Thus, UniPep provides access to human and mouse $N$-glycosylated proteins and their $N$-glycosylation sites for biomarker discovery. All the proteins including their protein ID are listed on this dynamic website. Furthermore, the website provides information on all these $N$-glycosylated proteins including identified $N$-glycosylated peptide sequences and probability scores.

Moreover, the consensus $N$-glycosylation sites of the proteins can be reached from this database. The database provides the in silico trysin digest of the proteins and the possible NXS/T motifs. Another bioinformatics tool in this website determines whether these glycosylation sites can be detected or not in an MS/MS experiment which is an important guide for the experimental design. As a next phase of the project, this library of theoretical peptides, which have already been scored for their likelihood of mass spec detection, will be compared to the experimentally deposited proteotypic peptides from a variety of LC/ MS/MS experiments.

\section{GlycoSuiteDB}

Unicarbkb (http://unicarbkb.org) provides information on both the glycan structure and glycosylated peptides of proteins [32,33]. This database includes all the published glycan types and glycosylation site information found throughout the literature from 1990 to 2005. Currently, there are 9436 entries from 864 references belonging to 245 species, including Homo sapiens, Rattus norvegicus and Mus musculus. On the website, proteins of interest can be searched by name, Uniprot, SwissProt or TrEMBL accession numbers. The database provides access to information such as the biological source of the protein, its glycosylation sites and possible glycan structures at those sites for both $\mathrm{N}$-glycosylated and $\mathrm{O}$-glycosylated proteins. Furthermore, it includes literature references and the relevant links to PubMed. The methods used for the identification of the glycans and glycosites are also provided on the website. Finally, glycoproteins associated with particular disease states in the literature are provided $[34,35]$. While a major disadvantage of GlycoSuiteDB is that it has not been updated since 2005, it was recently incorporated as part of UniCarbKB [33]. Since UniPep and GlycoSuiteDB are excellent sources for biomarker and therapeutics discovery, methods should be implemented to update and provide glycoproteomes of more organisms in addition to those currently catalogued.

\section{GlycoFly}

GlycoFly is another publicly available database for $N$ glycosylated proteins and peptides of Drosophila melanogaster [19]. Drosophila is an important model organism to study since it is often applied to interpret the effects of gene mutations on human diseases. For instance, a mutation in the volado/scab glycoprotein gene, which leads to glycan variations, has been shown to cause memory deficits [36] and a mutation of the wolknauel gene of the glycosylation pathway has resulted in disruptions in embryonic patterning [37]. Furthermore, blood nerve barrier dysfunction and loss of glial septate junctions in the peripheral nervous system have been observed when contactin, neuroglian, and neuroxin IV genes are mutated [38]. These proteins are highly glycosylated and localized to the nervous system of flies [19]. As a result, GlycoFly has focused on glycoproteome identification of the central nervous system of flies. Four hundred and seventy seven central nervous system glycoproteins containing 740 NXS/T glycosylation sites were identified. This information is available publicly on the GlycoFly website (http://betenbaugh.org/GlycoFly/) [39]. The proteins are listed with their Flybase IDs, and a specific protein of interest can be searched by name or sequence. The function of each protein, identified glycosylated peptide sequence and its probability are compiled as well. An example output from the website 
is displayed in Figure 1. The relative publications and an overview of the experiments as well as in-silico prediction tools and links to other glycoproteome databases are not yet active in this database.

\section{GlycoFish}

Danio rerio (zebrafish) is a promising model system to understand vertebrate development and human disease because of biological and functional similarities between humans and zebrafish. Larval and embryonic zebrafish have also been used to explore potential therapeutics for developmental disorders since some pharmacological agents, especially neurotoxins and neuroprotectants, have shown similar effects in zebrafish and humans [40,41]. Furthermore, mutations in zebrafish cause diseases that resemble human diseases; for example, both adult and embryo zebrafish have been used to understand neurological and neuromuscular diseases such as Huntington's, Alzheimer's and Parkinson's. [42-44]. Therefore, the glycoproteome of zebrafish embryos was characterized by our group in order to determine $N$-glycosylated sites of proteins present during in vertebrate development [20].

Using the hydrazide chemistry method, $169 \mathrm{~N}$-glycosylated proteins were identified. These proteins include $269 N$-glycosylation sites found on $265 N$-glycopeptides. In order to make this data publicly available, the GlycoFish database (http://betenbaugh.org/GlycoFish/), which [45] lists the mass spectrometer properties of identified $N$ glycopeptides and gives functional and sequential information on the identified $N$-glycosylated proteins, has been established. This database can be further improved by in-silico prediction of glycosylation sites as well as addition of related publications, overview of the experiment, and links to the other glycoproteomic databases.

\section{GlycoProtDB (GPDB)}

GlycoProtDB (http://jcggdb.jp/rcmg/gpdb/index.action) is a database for the $\mathrm{N}$-glycoproteins of Caenorhabditis elegans N2 and mouse tissues, identified from lectin chromatography experiments $[46,47]$. In order to enrich the $N$ glycosylated proteins, lectin affinity column based isotope coded glycosylation site specific tagging (IGOT) was used. The proteins were digested, applied to a lectin affinity column in order to enrich the $N$-glycosylated proteins, and $N$-glycanase treatment was performed to remove the glycosylated peptides in ${ }^{18} \mathrm{O}$-labeled water for tagging of the asparagine sites converted aspartate sites [48-50]. Then shotgun analysis with LC/MS/MS identified $400 \mathrm{~N}$-glycosites on 250 glycoproteins using this elegant technique in the initial study [48]. These numbers were increased to $1465 \mathrm{~N}$-glycosylated sites on 829 proteins in subsequent studies [50]. Furthermore, 1200 mouse liver glycoproteins, accessible in the GlycoProtDB database [49] were also identified using 2D-LCMS/MS studies.

Proteins of interest can be searched on GlycoProtDB by their name, amino acid length, molecular weight or database identifiers. A user friendly website provides information on the glycoprotein ID, amino acid sequence, and experimentally identified glycosylation sites of the proteins. It also provides access to the method and lectins used for the identification of these glycopeptides [46,47].

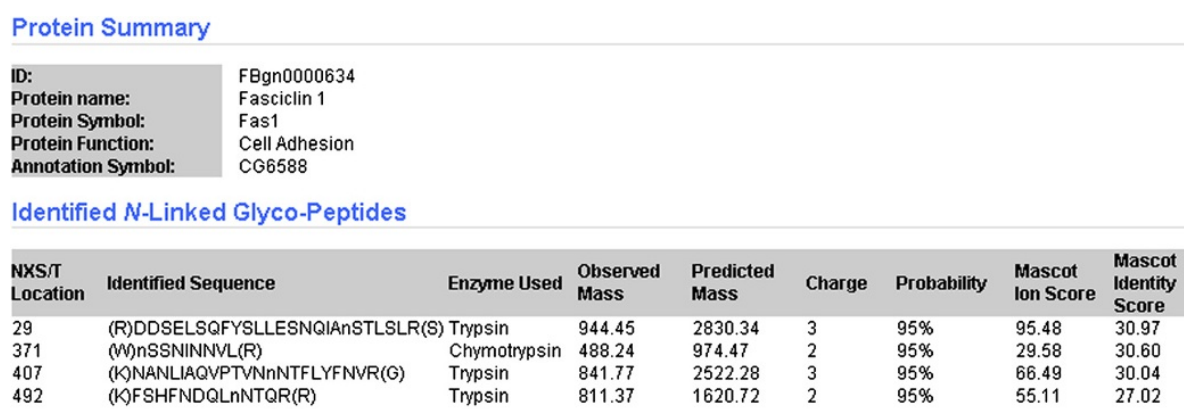

Protein Sequence

mlnaaa 11 lallcaanaaaaadladk 1 [rddse lsqfys l lesnqianst ls lrs] ct if vptneafqryksktahv lyhit teayt qkr l pntvssdmagnpplyitknsngdifvnna riips lsvetnsdgkrqimhi idev lep ltvkaghsdt pnnpnalkf lknaeefnvdnig vrtyrsqvtmakkesvydaagqht flvpvdegfklsarss lvdgkvidghvipntvifta aaqhddpkasaafedl 1 kvtvsffkqkngkmyvksnt ivgdakhrvgvv lae ivkanipv sngvvhlihrplmi idt tvtqf lqsfkfmnenaengalrkf yevimdnggavlddins 1 t evt i lapsnea [ wnssninnvlr] drnkarq q i nmhi ikdr lnvdkirq [ knanl iaqvp tvnnnt flyfnvrg] egsdtvi tvegggvnatvigadvagtngyvh $i$ idhv lovpyt tv 1 gklesdpmmsdt ykmg[kfshfndlinntorr] ft ffvprdkgwokteldypsahlicl gklesdpho adfsyhsksilerhlaisdkeytmkdlvkfsqesgovilptfrdslsirveeeagryvi wnykk invyrpdvectngi i hvidyp l leekdvvvaggsy lpessici i lanlimitvak
fln

Figure 1 Example of GlycoFly website protein, Fascilin 1 (http://betenbaugh.org/GlycoFly/). 


\section{O-GlycBase}

O-GlycBase (http://www.cbs.dtu.dk/databases/OGLYCBASE/) is a prediction website of the Technical University of Denmark (DTU) [51,52]. This database includes 242 proteins with $2413 \mathrm{O}$-glycosylation sites and relevant references. $\mathrm{O}$-glycosylated proteins were documented to establish a network for predicting the $O$-GalNac sites of the proteins [53]. This prediction database for the mucin-type $O$-glycosylated proteins is named NetOGlyc (www.cbs.dtu.dk/services/NetOGlyc/) [54], which identifies potential $O$-glycosylation sites for any submitted protein with $76 \%$ confidence $[53,54]$. Furthermore recently NetOGlyc4.0 model has been developed which is based on the first $O$-glycoproteome map of human consisting of $3000 \mathrm{O}$-glycosites from over $600 \mathrm{O}$-glycoproteins using genetic engineering approach [55-57]. O-Unique (http://www.cbs.dtu.dk/ftp/Oglyc/O-Unique.seq), another database established by DTU, includes 53 mucin type mammalian glycoproteins with 265 experimentally proven $O$-glycosylation sites [58].

\section{dbOGAP}

$O$-GlcNAcylation is the addition of $\beta$ - $\mathrm{N}$-acetylglucosamine (GlcNac) to Ser or Thr aminoacids by the O-GlcNac transferase (OGT) enzyme. Unlike mucin type $O$-glycosylation, GlcNAc attachment occurs only for nuclear and cytoplasmic proteins with no further addition or extension of carbohydrates. O-GlcNAcylation plays an important role in biological processes and has been associated with diseases such as diabetes, cancer, and neurodegeneration. For this reason, dbOGAP (http://cbsb.lombardi.georgetown.edu/ OGAP.html) database for O-GlcNAcylated proteins and sites was established and a support vector machine (SVM) based sequence program to predict the protein O-GlcNAcylation sites was developed [59]. This database includes 798 experimentally proved and 365 predicted proteins of human, rat, mouse, frog and fly [60]. For each protein entry, the experimentally characterized or predicted $\mathrm{O}$-GlcNacylation and phosphorylation sites are available at this website, along with the molecular and biological function of each protein and its importance in disease states. The $O$-GlcNAcScan feature allows users to predict $O$-GlcNacylation sites for any submitted protein $[59,60]$.

\section{Glycomic databases}

Both the glycosylation sites and the bound glycan structures represent important aspects of systems glycobiology. More than 200 glycosyltransferases are responsible for the addition and modification of carbohydrates with different linkages in order to generate a wide range of diverse glycans [61]. As a result, glycan characterization can be challenging due to the heterogeneity and complexity of oligosaccharide moieties. However, specific carbohydrates can play key roles in cell-cell recognition, receptor-ligand binding, protein interactions, and protein stability in vivo [62]. In recent years, high-throughput glycomic techniques have enabled fast and robust glycan characterization to demonstrate lot-to-lot consistency in pharmaceutical therapeutics and to understand the role of glycans in human disease [62].

Complete glycan profiling can include the detection, identification, and quantification of the carbohydrates as well as the the identification of linkages between specific monosaccharides. Different methods including chromatographic separation and mass spectrometry [63] are used for the analysis of glycans. Glycan analysis from a biological sample requires the release of an intact glycan from the protein followed by separation and detection using chromatography or mass spectrometry based glycan methods. Various combinations of methods are also used in glycan isolation and characterization as summarized in recent articles [62-72].

Evaluating glycans can represent a more complex task than proteomics or genomics because of the multiple glycosyltransfers that occur during glycan biosynthesis. Furthermore, various $O$ and $\mathrm{N}$-glycan structures are possible depending on the specific target proteins and glycosyltransferases present, making decoding the glycans challenging $[63,73]$. To enhance knowledge of glycomic patterns, glycomic databases are being established that document the different glycan structures and make this information publically available [73]. A table summarizing the various databases primarily concerned with glycomic studies is listed below.

\section{Consortium Functional Glycomics (CFG) glycan structure database}

CFG provides one of the largest databases for understanding the roles of carbohydrates in cell communication [28]. It also includes a glycan structural database (http://www.functionalglycomics.org/glycomics/molecule/ jsp/carbohydrate/carbMoleculeHome.jsp) in order to compile and integrate glycomic data sets for the glycoscience community [74]. CFG has provided both core facilities for data generation and a bioinformatics platform for annotating glycan structural data [75]. The analytical glycotechnology core facility of CFG has profiled permethylated $N$ - and $O$ - glycans for human and mouse tissues and cell lines. In addition, CarbBank and Glycominds, which include $N$ - and $O$-glycans analyzed in other studies, are integrated in this database. Different options to search for glycans of interest include their name, composition, molecular weight, Glycan ID, IUPAC ID, the cell line or tissue sample. Both basic and complex searches can be performed depending on the bioinformatics goals. For example, one can search for glycans containing sialic acid or those associated with human cancer. 
When selecting the glycan of interest, the glycan cartoon and IUPAC 2D structures are shown and its properties, such as molecular weight, are listed. Furthermore, CFG identifies whether this glycan is $\mathrm{N}$ - or $\mathrm{O}$-linked and studies related to this glycan are noted in the reference section $[74,75]$. The substructure search option is another uncommon and useful feature of CFG database. The substructure interface provides different common carbohydrate motifs, for $O$-linked and $N$-linked glycans that can be modified or extended to form the desired glycan structure [74,75].

\section{GlycoBase}

Fluorophore labeling using 2-aminobenzamide (2-AB) is often used for labeling the glycans for subsequent HPLC analysis. A 2-AB labeled dextran ladder was used to assign glucose unit (GU) values based on the retention times of glycans [76]. GU values representing the HPLC retention times for more than 350 glycan structures are available on the GlycoBase database (http://glycobase.nibrt. ie/glycobase/show_nibrt.action) [77]. In addition to the GU values, monosaccharide compositions and their linkages are represented with pictures for each glycan. Each entry has links for the exoglycosidase digestion products and the groups where the glycan of interest can be found. Also, relevant publications related to these glycans are listed as references [76,78].

GlycoBase also includes the GlycoExtractor interface for extraction of HPLC glycan data into a common format [79]. GlycoExtractor can export the peak areas and GU values from large sets of HPLC data in order to integrate shared data in the same format. This format makes data analysis and storage easier for glycan profiling, which is helpful for biomarker discovery and generation of therapeutics [80].

\section{GlycomeDB}

GlycomeDB is a database established for the integration of the carbohydrate structures and annotations from seven different publicly available databases (CFG, Bacterial Carbohydrate Structure Database (BCSDB), GLYCOSCIENCES.de, Kyoto Encyclopedia of Genes and Genomes (KEGG), EUROCarbDB and Carbbank) [81]. GlycomeDB also introduced both GlycoCT and GlycoUpdateDB interfaces. GlcyoCT is a universal data format established for the incorporation of glycan datasets onto the GlycomeDB website. GlycoUpdateDB interface generates updates from different databases to the website on a weekly basis. After downloading the datasets from public databases, GlycoUpdateDB translates the data into the GlycoCT format and integrates the new data into GlycomeDB website. More than 35,873 different carbohydrate sequences have been uploaded in GlycoCT format with 11,822 structures fully determined including all linkage positions, base type, anomers, ring size and modifications $[82,83]$. GlycomeDB provides the image of the glycan structure, its specifications in GlycoCT format, and links to the external databases for further information on the glycan of interest. It is also possible to learn all the identified oligosaccharide structures for a particular species. When searching a specific species, the website lists the glycans with their cartoon representations and references [81].

GlycomeDB has also absorbed another important database: the Japan Consortium Glycobiology and Glycotechnology Database (JCGGDB) (http://jcggdb.jp/index_en. $\mathrm{html}$ ), which itself is composed of the GlycoGene Database (GGDB) (http://jcggdb.jp/rcmg/ggdb/) and Glycan Mass Spectral Database (GMDB) (http://jcggdb.jp/ rcmg/glycodb/Ms_ResultSearch) [84-86]. The JCGGDB database provides a different approach for displaying glycomic information compared to other available databases.

GGDB includes all the identified genes related with a glycosylation pathway such as glycosyltransferases, sialyltransferases, carbohydrate transporters and synthases. All the DNA and mRNA sequences of these enzymes with their gene expression profiles in tissues are included as well. Furthermore, graphical representations of the substrate specificities are also provided [84]. The GMDB approach is similar to the GlycoBase approach for the identification of glycans. However, instead of GU values, GMDB provides spectral view of glycans obtained with MALDI-QIT-TOF MS. Each carbohydrate structure has an $\mathrm{MS}^{\mathrm{n}}$ fragmentation pattern and these collisioninduced dissociation spectra are stored in the database to enable spectral matching and glycan identification. The $\mathrm{MS}^{\mathrm{n}}$ spectra of any glycan can then be searched based on its $\mathrm{m} / \mathrm{z}$ value or composition. The website also provides an option to include modifications such as phosphorylation on the glycan of interest. If the glycan is coupled with a fluorescent reagent, such as 2aminopyridine, this can also be included in the list of labeling groups to look for the specific spectra of 2 -aminopyridine coupled glycans $[85,86]$.

\section{GlycoSuiteDB}

In addition to being a glycoproteome database, GlycoSuiteDB, established by Tyrian Diagnostics Ltd provides access tomore than 3238 unique carbohydrate structures from 245 different species. GlycoSuiteDB is a web-friendly database which provides information on the mass and composition of the glycan, the linkages and the anomeric configuration. This database gives detailed information on the cell line or tissue in which each glycan structure is found, as well as the method used to determine the specified glycan, its role in disease states or therapeutic production, and links to references [32,34,35].

The website also lists all the available glycan types in the database with a particular composition or mass. In 
addition, one can construct or extend a structure and then look up if that specific carbohydrate has been identified or investigated in the literature. Another search option available is the ability to find glycans associated with a specific biological source or disease. For example, when performing a search with blood as your biological source, 49 different glycans are specified [32].

\section{EuroCarbDB}

EUROCarbDB (https://code.google.com/p/eurocarb/) is a European based core database for the collection of carbohydrate data and the development and housing of corresponding bioinformatics tools [87]. This initiative has been established to provide the technical infrastructure needed for standardization of the glycomic data and the appropriate analytical tools. EuroCarbDB aims to compile large, high quality primary research data sets from MS, NMR and HPLC experimental work into a single location in order to create common standards for storing these datasets. In conjunction, EuroCarbDB has established bioinformatic tools for analyzing, processing and identifying the glycan structures from MS, NMR spectra and HPLC profiles. For example, a software tool has been developed, GlycanBuilder, which can be used to visualize, display and assemble glycan structures with a symbolic notation. GlycanBuilder can either be used in a user-independent manner to display glycans or as a userdependent tool to draw specific glycan structures [88]. In addition, GlycoWorkbench is another glycoinformatics tool which can be used to annotate the $N$ and $O$-glycans from mass spectra data [89]. One of the challenges in glycomics databases has been the digital representation of carbohydrate structures in a computer readable format. Two glycobioinformatics tools, Glyco-CT and Glyde have been established for encoding the glycan structures. Recently Glyde has been recognized as the standard format for the exchange of information between databases [88]. Besides these, Glyde II and Glyde II DTD were developed by University of Georgia. Glyde II DTD especially provides the preservation of partonomy and granularity in the carbohydrates [90].

\section{Databases for Glycan-protein interactions}

Glycan-Binding Proteins (GBP) such as antibodies, lectins, and receptors has been used for glycan recognition over many years. However, determination of specificities of GBPs required a large amount of the glycans and much labor-intensive preparation prior to the development of glycan microarray technology. Glycan microarray technology has since accelerated studies in glycomics since glycan binding specificities can be analyzed quantitatively in a short period of time using much smaller amounts of sample material [91].
The most widely used highthroughput method for glycan profiling are lectin microarrays, which can analyze multiple lectin-glycan interactions simultaneously [92-94]. Antibodies are also used in glycan microarrays since they can be specific to particular carbohydrate epitopes. Antigenic epitopes such as Lewis $\mathrm{x}$ and Sialyl Lewis A can be strongly recognized by specific monoclonal antibodies $[73,91,95]$. However, antibodies are usually unable to differentiate between $\mathrm{O}$-glycans, $\mathrm{N}$-glycans or glicolipids. They typically bind to their specific epitopes regardless of the glycan type [95]. The methods that are used in glycan microarrays and available databases are discussed below.

\section{CFG}

The Consortium for Functional Glycomics (CFG) group also has a Protein-Carbohydrate Interaction Core facility which applies two different methodologies for protein analysis and glycan recognition. Both microwell based and glass slide arrays similar to DNA microarrays are used to screen hundreds of glycans, lectins, antibodies and pathogenic proteins. Streptavidin-coated wells are covered with biotinylated synthetic or biological glycans to identify novel carbohydrate binding ligands. Moreover, glycan printing on the $N$-hydroxysuccinimide-reacted glass slide arrays is being used to expand number of possible glycan ligand targets. This technology also has an advantageous low signal to noise ratio [74].

The CFG database allows users to search through plate, printed and pathogen arrays for the specific analyte of interest. Numerous animal lectins such as C-type lectins, siglecs, galectins as well as plant lectins, pathogens, microbial lectins, antibodies, serum, cells and organisms are available under the analyte category. When the analyte of interest and array type are chosen, the website finds all the studies related to them. The primary glycan binding specificity, ligand site and any information related to this glycan binding protein are also provided in the database $[96,97]$.

\section{LfDB}

The Lectin Frontier Database (LfDB) was established by JCGGDB and provides quantitative information on glycanprotein interactions. The binding specificity of each lectin to different glycans is variable and this affinity can be quantified in terms of an association constant $\left(\mathrm{K}_{\mathrm{a}}\right)$. Frontal affinity chromatography with fluorescence detection (FAC-FD) is a common method used to determine affinity constants since it produces reliable and reproducible data [47]. As shown in Figure 2, Langmuir's adsorption principle is applied in this isocratic elution system.

Pyridylaminated glycans (PA-glycans) in low concentrations can be loaded onto the lectin-immobilized column, and the binding specificity of a glycan calculated based on 


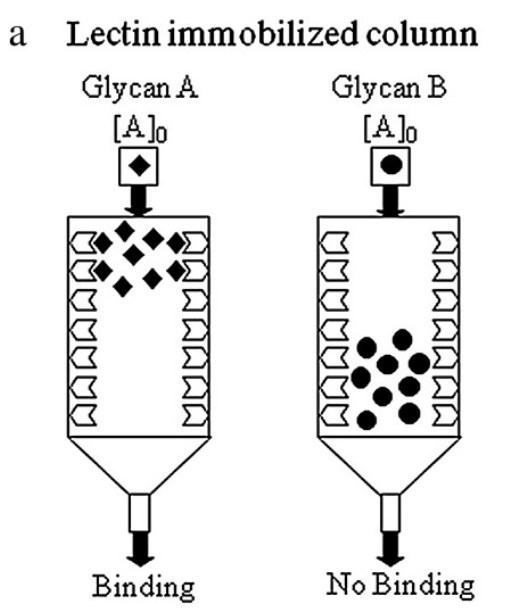

b

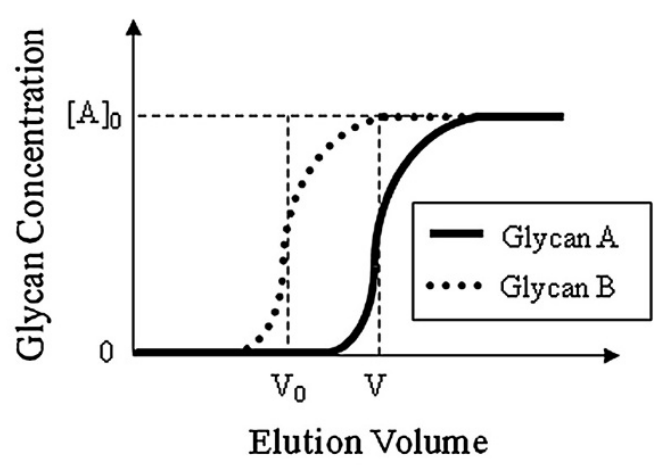

Figure 2 Frontal affinity chromatography for the quantification of lectin-glycan constants. Schematic graphs of a) lectin immobilized column b) isocratic elution system.

the change in the volumes as shown in the following equations.

$$
K_{d}=\frac{B_{t}}{\left(V-V_{0}\right)}-\left[A_{0}\right] \text { and } K_{a}=1 / K_{d}
$$

Where $K_{a}$ is the affinity constant, $B_{t}$ is the effective lectin content, $\left[\mathrm{A}_{0}\right]$ is the initial glycan concentration, and $\mathrm{V}-\mathrm{V}_{0}$ is the difference between the initial glycan volume of the glycan of interest and a negative control [92].

In LfDB (http://jcggdb.jp/rcmg/glycodb/LectinSearch), a variety of lectin affinities towards glycans are available. Any lectin type or monosaccharide specificity can be searched. Once the glycan binding protein is found, all the information related to this protein and its $K_{a}$ values toward different glycans can be obtained from this database [98].

\section{Conclusion}

Fifteen different glycomic and glycoproteomic related databases are described in the current study. These databases include more than 30,000 entries for experimentally identified or predicted glycans and glycopeptides. The structural information on the glycan or glycosite of these glycoproteins and hyperlinks to their references are also provided in these databases. Each of these databases has key features. For instance Unipep includes both experimentally proven glycoproteins and their glycosites and also in-silico predicted glycosites on human proteins. GlycoFly focuses on the N-glycosylated peptides of Drosophila melanogaster whereas GlycoFish provides the list of N-glycosites of zebrafish. O-GlycBase, dbOGAP are the specific databases for $\mathrm{O}$-glycosylation and $\mathrm{O}$ GlcNAcylation. CFG and EuroCarbDB are the two largest databases for carbohydrates whereas GlycoBase and GlcyomeDB databases include extensive information on the glycans. Furthermore databases such as CFG and LFDB provide information on the glycan-protein and lectin interactions. This review will be a useful resource for glycobiology studies and institutions searching for information on glycoproteins of interest. Furthermore, assembling the databases in this review and others will assist in the eventual formation of a single resource for glycomic and glycoproteomics high-throughput data. In the long term, the glycobiology community should strive to create a fully integrated and dynamic database that includes all the elements described in this review. One vision would be a database that has all the glycosylated proteins, indicating if they are $\mathrm{O}$ or $\mathrm{N}$-glycosylated, and showing their $\mathrm{O}$ and $\mathrm{N}$-glycosylation sites. We could then add additional functionalities to the database including all known glycan structures obtained at the designated glycosylation site together with specific glycosylation linkages. Of course, some of this data are not yet available, and thus there are additional experimental data and complementary bioinformatics that need to be obtained before a comprehensive glycomics database can become a reality.

\section{Competing interests}

The authors declare that they have no competing interests.

\section{Authors' contributions}

DBH has done the literature search and drafted the review article. DW, JC and $E J$ have done extensive research for the collection of glycoproteomic databases and glycomic databases. They have worked on the figures and tables. YT has done research and has provided the drafting of the analytical methods used for glycoproteome identification. DW, SSK, MB and HZ edited and further improved the draft for publication. All authors read and approved the final manuscript.

\section{Author details}

'Department of Chemical and Biomolecular Engineering, Johns Hopkins University, Baltimore, MD, USA. ${ }^{2}$ Department of Pathology, Johns Hopkins University, Baltimore, MD, USA. ${ }^{3}$ Department of Biochemistry and Molecular Biology, Johns Hopkins Bloomberg School of Public Health, Baltimore, MD, USA. 
Received: 1 October 2013 Accepted: 20 February 2014

Published: 13 April 2014

\section{References}

1. Shental-Bechor D, Levy Y: Effect of glycosylation on protein folding: a close look at thermodynamic stabilization. Proc Natl Acad Sci U S A 2008 105:8256-8261.

2. Sola RJ, Griebenow K: Glycosylation of therapeutic proteins: an effective strategy to optimize efficacy. BioDrugs 2010, 24:9-21.

3. Zhu J, Wang Y, Yu Y, Wang Z, Zhu T, Xu X, Liu H, Hawke D, Zhou D, Li Y: Aberrant fucosylation of glycosphingolipids in human hepatocellular carcinoma tissues. Liver Int 2013, 34(1):147-160.

4. Tian Y, Zhang H: Glycoproteomics and clinical applications. Proteomics Clin Appl 2010, 4:124-132.

5. Ahn YH, Shin PM, Kim YS, Oh NR, Ji ES, Kim KH, Lee YJ, Kim SH, Yoo JS: Quantitative analysis of aberrant protein glycosylation in liver cancer plasma by AAL-enrichment and MRM mass spectrometry. Analyst 2013, 138(21):6454-6462.

6. Jaeken J: Congenital disorders of glycosylation (CDG): it's (nearly) all in it! J Inherit Metab Dis 2011, 34:853-858.

7. Xiong $L$, Andrews D, Regnier F: Comparative proteomics of glycoproteins based on lectin selection and isotope coding. J Proteome Res 2003, 2:618-625.

8. Nilsson J, Ruetschi U, Halim A, Hesse C, Carlsohn E, Brinkmalm G, Larson G: Enrichment of glycopeptides for glycan structure and attachment site identification. Nat Methods 2009, 6:809-811.

9. Aspberg K, Porath J: Group-specific adsorption of glycoproteins. Acta Chem Scand 1970, 24:1839-1841.

10. Lloyd KO: The preparation of two insoluble forms of the phytohemagglutinin, concanavalin $A$, and their interactions with polysaccharides and glycoproteins. Arch Biochem Biophys 1970 137:460-468.

11. Kim EH, Misek DE: Glycoproteomics-based identification of cancer biomarkers. Int J Proteomics 2011, 2011:601937.

12. Pan $\mathrm{S}$, Chen R, Aebersold R, Brentnall TA: Mass spectrometry based glycoproteomics-from a proteomics perspective. Mol Cell Proteomics 2011, 10:R110 003251. doi:10.1074/mcp.R110.003251.

13. Jun-ichi Furukawa NFYS: Recent advances in cellular glycomic analyses. Biomolecules 2013, 3(1):198-225

14. Rillahan CD, Paulson JC: Glycan microarrays for decoding the glycome. Annu Rev Biochem 2011, 80:797-823.

15. Rakus JF, Mahal LK: New technologies for glycomic analysis: toward a systematic understanding of the glycome. Annu Rev Anal Chem (Palo Alto, Calif) 2011, 4:367-392.

16. Wada Y, Dell A, Haslam SM, Tissot B, Canis K, Azadi P, Backstrom M, Costello CE, Hansson GC, Hiki Y, Ishihara M, Ito H, Kakehi K, Karlsson N, Hayes CE, Kato K, Kawasaki N, Khoo KH, Kobayashi K, Kolarich D, Kondo A, Lebrilla C, Nakano M, Narimatsu H, Novak J, Novotny MV, Ohno E, Packer NH, Palaima E, Renfrow MB, et al: Comparison of methods for profiling o-glycosylation human proteome organisation human disease glycomics/proteome initiative multi-institutional study of iga1. Mol Cell Proteomics 2010, 9:719-727.

17. Wada Y, Azadi P, Costello CE, Dell A, Dwek RA, Geyer H, Geyer R, Kakehi K, Karlsson NG, Kato K, Kawasaki N, Khoo KH, Kim S, Kondo A, Lattova E, Mechref Y, Miyoshi E, Nakamura K, Narimatsu H, Novotny MV, Packer NH, Perreault H, Peter-Katalinic J, Pohlentz G, Reinhold VN, Rudd PM, Suzuki A, Taniguchi N: Comparison of the methods for profiling glycoprotein glycans - HUPO human disease glycomics/proteome initiative multiinstitutional study. Glycobiology 2007, 17:411-422.

18. Zhang H, Loriaux P, Eng J, Campbell D, Keller A, Moss P, Bonneau R, Zhang N, Zhou Y, Wollscheid B, Cooke K, Yi EC, Lee H, Peskind ER, Zhang J, Smith RD, Aebersold R: UniPep - a database for human N-linked glycosites: a resource for biomarker discovery. Genome Biol 2006, 7:8:R73.1:R73.11.

19. Baycin-Hizal D, Tian Y, Akan I, Jacobson E, Clark D, Chu J, Palter K, Zhang H, Betenbaugh MJ: GlycoFly: a database of Drosophila N-linked glycoproteins identified using SPEG-MS techniques. J Proteome Res 2011, 10:2777-2784

20. Baycin-Hizal D, Tian Y, Akan I, Jacobson E, Clark D, Wu A, Jampol R, Palter K, Betenbaugh M, Zhang H: GlycoFish: a database of zebrafish N-linked glycoproteins identified using SPEG method coupled with LC/MS. Anal Chem 2011, 83:5296-5303.
21. Zhang $Y$, Yin $H$, Lu H: Recent progress in quantitative glycoproteomics. Glycoconj J 2012, 5-6:249-258.

22. Nwosu CC, Seipert RR, Strum JS, Hua SS, An HJ, Zivkovic AM, German BJ, Lebrilla CB: Simultaneous and extensive site-specific N- and O-glycosylation analysis in protein mixtures. J Proteome Res 2011, 10:2612-2624.

23. Wang Y, Wu SL, Hancock WS: Approaches to the study of N-linked glycoproteins in human plasma using lectin affinity chromatography and nano-HPLC coupled to electrospray linear ion trap-Fourier transform mass spectrometry. Glycobiology 2006, 16:514-523.

24. Zhang H, Li XJ, Martin DB, Aebersold R: Identification and quantification of $\mathrm{N}$-linked glycoproteins using hydrazide chemistry, stable isotope labeling and mass spectrometry. Nat Biotechnol 2003, 21:660-666.

25. Pan S, Tamura Y, Chen R, May D, McIntosh MW, Brentnall TA: Large-scale quantitative glycoproteomics analysis of site-specific glycosylation occupancy. Mol Biosyst 2012, 8:2850-2856

26. Sanda M, Pompach P, Brnakova Z, Wu J, Makambi K, Goldman R: Quantitative liquid chromatography-mass spectrometry-multiple reaction monitoring (LC-MS-MRM) analysis of site-specific glycoforms of haptoglobin in liver disease. Mol Cell Proteomics 2013, 12:1294-1305.

27. UNIPEP. www.unipep.org.

28. Functional Glycomics Gateway. http://www.functionalglycomics.org/.

29. Tian Y, Kelly-Spratt KS, Kemp CJ, Zhang H: Identification of glycoproteins from mouse skin tumors and plasma. Clin Proteomics 2008, 4:117-136.

30. Tian Y, Kelly-Spratt KS, Kemp CJ, Zhang H: Mapping tissue-specific expression of extracellular proteins using systematic glycoproteomic analysis of different mouse tissues. J Proteome Res 2010, 9:5837-5847.

31. Zielinska DF, Gnad F, Wisniewski JR, Mann M: Precision mapping of an in vivo $\mathrm{N}$-glycoproteome reveals rigid topological and sequence constraints. Cell 2010, 141:897-907.

32. GlycoSuiteDB. http://glycosuitedb.expasy.org/.

33. UnicarbBKB. http://unicarbkb.org

34. Cooper CA, Harrison MJ, Wilkins MR, Packer NH: GlycoSuiteDB: a new curated relational database of glycoprotein glycan structures and their biological sources. Nucleic Acids Res 2001, 29:332-335.

35. Cooper CA, Joshi HJ, Harrison MJ, Wilkins MR, Packer NH: GlycoSuiteDB: a curated relational database of glycoprotein glycan structures and their biological sources. 2003 update. Nucleic Acids Res 2003, 31:511-513.

36. Grotewiel MS, Beck CDO, Wu KH, Zhu XR, Davis RL: Integrin-mediated short-term memory in Drosophila. Nature 1998, 391:455-460.

37. Haecker A, Bergman M, Neupert C, Moussian B, Luschnig S, Aebi M, Mannervik M: Wollknauel is required for embryo patterning and encodes the Drosophila ALG5 UDP-glucose: dolichyl-phosphate glucosyltransferase. Development 2008, 135:1745-1749.

38. Banerjee S, Pillai AM, Paik R, Li JJ, Bhat MA: Axonal ensheathment and septate junction formation in the peripheral nervous system of Drosophila. J Neurosci 2006, 26:3319-3329.

39. GlycoFly database. http://betenbaugh.org/GlycoFly/

40. Guo S: Using zebrafish to assess the impact of drugs on neural development and function. Expert Opin Drug Deliv 2009, 4:715-726.

41. Pichler FB, Laurenson S, Williams LC, Dodd A, Copp BR, Love DR: Chemical discovery and global gene expression analysis in zebrafish. Nat Biotechnol 2003, 21:879-883.

42. Leimer U, Lun K, Romig H, Walter J, Grunberg J, Brand M, Haass C: Zebrafish (Danio rerio) presenilin promotes aberrant amyloid beta-peptide production and requires a critical aspartate residue for its function in amyloidogenesis. Biochemistry 1999, 38:13602-13609.

43. Son OL, Kim HT, Ji MH, Yoo KW, Rhee M, Kim CH: Cloning and expression analysis of a Parkinson's disease gene, uch-L1, and its promoter in zebrafish. Biochem Biophys Res Commun 2003, 312:601-607.

44. Karlovich CA, John RM, Ramirez L, Stainier DYR, Myers RM: Characterization of the Huntington's disease (HD) gene homolog in the zebrafish Danio rerio. Gene 1998, 217:117-125.

45. GlycoFish database. http://betenbaugh.org/GlycoFish/.

46. GlycoDB. http://jcggdb.jp/rcmg/glycodb/.

47. Narimatsu H, Sawaki $H$, Kuno A, Kaji H, Ito H, Ikehara Y: A strategy for discovery of cancer glyco-biomarkers in serum using newly developed technologies for glycoproteomics. Febs Journal 2010, 277:95-105.

48. Kaji H, Saito H, Yamauchi Y, Shinkawa T, Taoka M, Hirabayashi J, Kasai K, Takahashi N, Isobe T: Lectin affinity capture, isotope-coded tagging and mass spectrometry to identify N-linked glycoproteins. Nat Biotechnol 2003, 21:667-672. 
49. Kaji H, Yamauchi $Y$, Takahashi N, Isobe T: Mass spectrometric identification of N-linked glycopeptides using lectin-mediated affinity capture and glycosylation site-specific stable isotope tagging. Nat Protoc 2006, 1:3019-3027.

50. Kaji H, Kamiie J-i, Kawakami H, Kido K, Yamauchi Y, Shinkawa T, Taoka M, Takahashi N, Isobe T: Proteomics reveals N-linked glycoprotein diversity in Caenorhabditis elegans and suggests an atypical translocation mechanism for integral membrane proteins. Mol Cell Proteomics 2007, 6:2100-2109.

51. O-GlycBase. http://www.cbs.dtu.dk/databases/OGLYCBASE/.

52. Gupta R, Birch H, Rapacki K, Brunak S, Hansen JE: O-glycbase version 4.0: a revised database of o-glycosylated proteins. Nucleic Acids Res 1999, 27:370-372.

53. Julenius K, Molgaard A, Gupta R, Brunak S: Prediction, conservation analysis, and structural characterization of mammalian mucin-type O-glycosylation sites. Glycobiology 2005, 15:153-164.

54. NetOGlyc. www.cbs.dtu.dk/services/NetOGlyc/.

55. Steentoft C, Vakhrushev SY, Vester-Christensen MB, Schjoldager KT, Kong Y, Bennett EP, Mandel U, Wandall H, Levery SB, Clausen H: Mining the O-glycoproteome using zinc-finger nuclease-glycoengineered SimpleCell lines. Nat Methods 2011, 8:977-982.

56. Steentoft C, Vakhrushev SY, Joshi HJ, Kong Y, Vester-Christensen MB, Schjoldager KT, Lavrsen K, Dabelsteen S, Pedersen NB, Marcos-Silva L, Gupta R, Bennett EP, Mandel U, Brunak S, Wandall HH, Levery SB, Clausen H: Precision mapping of the human O-GalNAc glycoproteome through SimpleCell technology. EMBO J 2013, 32:1478-1488.

57. Steentoft C, Bennett EP, Clausen H: Glycoengineering of human cell lines using zinc finger nuclease gene targeting: SimpleCells with homogeneous GalNAc O-glycosylation allow isolation of the O-glycoproteome by one-step lectin affinity chromatography. Methods Mol Biol 2013, 1022:387-402

58. O-Glycbase: O-Unique. http://www.cbs.dtu.dk/ftp/Oglyc/O-Unique.seq

59. dbOGAP. http://cbsb.lombardi.georgetown.edu/OGAP.html.

60. Wang J, Torii M, Liu H, Hart GW, Hu Z-Z: DbOGAP - an integrated bioinformatics resource for protein O-GICNAcylation. BMC bioinformatics 2011, 12:91. doi:10.1186/1471-2105-12-91.

61. Hua S, Nwosu CC, Strum JS, Seipert RR, An HJ, Zivkovic AM, German JB, Lebrilla CB: Site-specific protein glycosylation analysis with glycan isomer differentiation. Anal Bioanal Chem 2012, 403:1291-1302.

62. Szabo Z, Guttman A, Rejtar T, Karger BL: Improved sample preparation method for glycan analysis of glycoproteins by CE-LIF and CE-MS Electrophoresis 2010, 31:1389-1395.

63. Pabst M, Altmann F: Glycan analysis by modern instrumental methods. Proteomics 2011, 11:631-643

64. Bereman MS, Young DD, Deiters A, Muddiman DC: Development of a robust and high throughput method for profiling $\mathrm{N}$-linked glycans derived from plasma glycoproteins by NanoLC-FTICR mass spectrometry. J Proteome Res 2009, 8:3764-3770.

65. Kronewitter SR, de Leoz ML, Peacock KS, McBride KR, An HJ, Miyamoto S, Leiserowitz GS, Lebrilla CB: Human serum processing and analysis methods for rapid and reproducible N-glycan mass profiling. J Proteome Res 2010, 9:4952-4959.

66. Palm AK, Novotny MV: A monolithic PNGase F enzyme microreacto enabling glycan mass mapping of glycoproteins by mass spectrometry. Rapid Commun Mass Spectrom 2005, 19:1730-1738.

67. Szabo Z, Guttman A, Karger BL: Rapid release of N-linked glycans from glycoproteins by pressure-cycling technology. Anal Chem 2010, 82:2588-2593.

68. Lonardi E, Balog Cl, Deelder AM, Wuhrer M: Natural glycan microarrays. Expert Rev Proteomics 2010, 7:761-774

69. Ruhaak LR, Zauner G, Huhn C, Bruggink C, Deelder AM, Wuhrer M: Glycan labeling strategies and their use in identification and quantification. Anal Bioanal Chem 2010, 397:3457-3481.

70. Reinhold V, Zhang H, Hanneman A, Ashline D: Toward a platform for comprehensive glycan sequencing. Mol Cell Proteomics 2013, 12:866-873.

71. Mechref Y, Hu Y, Desantos-Garcia JL, Hussein A, Tang H: Quantitative glycomics strategies. Mol Cell Proteomics 2013, 12:874-884.

72. Lazar IM, Lazar AC, Cortes DF, Kabulski JL: Recent advances in the MS analysis of glycoproteins: theoretical considerations. Electrophoresis 2011 32:3-13.
73. Comelli EM, Head SR, Gilmartin T, Whisenant T, Haslam SM, North SJ, Wong NK, Kudo T, Narimatsu H, Esko JD, Drickamer K, Dell A, Paulson JC: A focused microarray approach to functional glycomics: transcriptional regulation of the glycome. Glycobiology 2006, 16:117-131.

74. Glycan Structure Database. http://www.functionalglycomics.org/ glycomics/molecule/jsp/carbohydrate/carbMoleculeHome.jsp.

75. Raman R, Venkataraman M, Ramakrishnan S, Lang W, Raguram S, Sasisekharan R: Advancing glycomics: implementation strategies at the consortium for functional glycomics. Glycobiology 2006, 16:82R-90R.

76. Royle L, Radcliffe CM, Dwek RA, Rudd PM: Detailed structural analysis of Nglycans released from glycoproteins in SDS-PAGE gel bands using HPLC combined with exoglycosidase array digestions. In Methods in Molecular Biology. Volume 347. Edited by Brockhausen l.; 2006:125-143. Methods in Molecular Biology.

77. GlycoBase 3.2. http://glycobase.nibrt.ie/glycobase/show_nibrt.action

78. Campbell MP, Royle L, Radcliffe CM, Dwek RA, Rudd PM: GlycoBase and autoGU: tools for HPLC-based glycan analysis. Bioinformatics 2008, 24:1214-1216.

79. GlycoExtractor. http://glycobase.nibrt.ie/glycobase/browse_glycans.action.

80. Artemenko NV, Campbell MP, Rudd PM: GlycoExtractor: a Web-based interface for high throughput processing of HPLC-glycan data. J Proteome Res 2010, 9:2037-2041.

81. GlycomeDB. http://www.glycome-db.org/About.action.

82. Ranzinger R, Herget S, Wetter T, von der Lieth C-W: GlycomeDB - integration of open-access carbohydrate structure databases. BMC bioinformatics 2008, 9:384. doi:10.1186/1471-2105-9-384

83. Ranzinger R, Herget S, von der Lieth C-W, Frank M: GlycomeDB-a unified database for carbohydrate structures. Nucleic Acids Res 2011, 39:D373-D376.

84. GlycoGene Database. http://riodb.ibase.aist.go.jp/rcmg/ggdb/.

85. Glycan Mass Spectral Database. http://riodb.ibase.aist.go.jp/rcmg/glycodb/ Ms ResultSearch.

86. GlycoDB. http://riodb.ibase.aist.go.jp/rcmg/glycodb/Top.

87. EuroCarb DB. https://code.google.com/p/eurocarb/.

88. Ceroni A, Dell A, Haslam SM: The GlycanBuilder: a fast, intuitive and flexible software tool for building and displaying glycan structures. Source Code Biol Med 2007, 2:3.

89. Damerell D, Ceroni A, Maass K, Ranzinger R, Dell A, Haslam SM: The GlycanBuilder and GlycoWorkbench glycoinformatics tools: updates and new developments. Biol Chem 2012, 393:1357-1362.

90. Glyde-II Format. http://glycomics.ccrc.uga.edu/GLYDE-II/GLYDEdescription_v0.7.pdf

91. Heimburg-Molinaro J, Song X, Smith DF, Cummings RD: Preparation and analysis of glycan microarrays. In Curr Protoc Protein Sci. 2011. Chapter 12: Unit 12.10. Edited by Coligan JE, et al. ; 2011.

92. Hirabayashi J: Concept, strategy and realization of lectin-based glycan profiling. J Biochem 2008, 144:139-147.

93. Li Y, Tao S-C, Bova GS, Liu AY, Chan DW, Zhu H, Zhang H: Detection and verification of glycosylation patterns of glycoproteins from clinical specimens using lectin microarrays and lectin-based immunosorbent assays. Anal Chem 2011, 83:8509-8516.

94. Meany DL, Hackler L Jr, Zhang H, Chan DW: Tyramide signal amplification for antibody-overlay lectin microarray: a strategy to improve the sensitivity of targeted glycan profiling. J Proteome Res 2011, 10:1425-1431.

95. Varki A: Essentials of glycobiology. 1999

96. Functional Glycomics Gatewar Main page. http://www. functionalglycomics.org/glycomics/publicdata/primaryscreen.jsp.

97. Functional Glycomics Gateway Glycan Binding Protein. http://www functionalglycomics.org/glycomics/molecule/ssp/gbpMolecule-home.jsp.

98. Lectin Frontier Database. http://riodb.ibase.aist.go.jp/rcmg/glycodb/ LectinSearch.

doi:10.1186/1559-0275-11-15

Cite this article as: Baycin Hizal et al:: Glycoproteomic and glycomic databases. Clinical Proteomics 2014 11:15. 